\title{
Paper Enabling Power Beacons and Wireless Power Transfers for Non-Orthogonal Multiple Access Networks
}

\author{
Chi-Bao Le ${ }^{1}$ and Nhan Duc Nguyen ${ }^{2}$ \\ ${ }^{1}$ Faculty of Electronics Technology, Industrial University of Ho Chi Minh City (IUH), Ho Chi Minh City, Vietnam \\ 2 Innovation Center, Van Lang University, Ho Chi Minh City, Vietnam
}

https://doi.org/10.26636/jtit.2021.152421

\begin{abstract}
This paper studies downlink cellular networks relying on non-orthogonal multiple access (NOMA). Specifically, the access point (AP) is able to harvest wireless power from the power beacon (PB). In the context of an AP facilitated with multiple antennas, the transmit antenna selection procedure is performed to process the downlink signal, with the transmission guaranteed by energy harvesting. Therefore, a wireless power transfer-based network is introduced to overcome power outages at the AP. In particular, an energy-constrained AP harvests energy from the radio frequency signals transmitted by the PB in order to assist in transmitting user data. Outage performance and ergodic capacity are evaluated with the use of closed-form expressions. In order to highlight some insights, approximate computations are provided. Finally, numerical simulations are performed to confirm the benefits of combining the downlink NOMA transmission and the transmit power scheme at the AP in order to serve a multitude of users.
\end{abstract}

Keywords-ergodic capacity, NOMA, outage probability, power beacon.

\section{Introduction}

The main requirements faced by $5 \mathrm{G}$ systems include the following: high demand for data-intensive services and improved bandwidth availability for cellular networks [1], [2]. For multiple accesses (MA) techniques in 5G, some non-orthogonal techniques such as power domain nonorthogonal multiple accesses (PD-NOMA) [3] and sparse code multiple accesses (SCMA) [4], are introduced. In the context of NOMA, PD-NOMA allocates a sub-carrier to multiple users at the same time, by employing superimposed coding on the transmitter side, while user signals are detected thanks to the successive interference cancellation (SIC) method adopted at the receiver side. In SCMA, each sub-carrier may be implemented by allocating different codebooks on the transmitter side, while simultaneously applying the message passing algorithm (MPA) for the receiver side to detect user signals.

PD-NOMA and SCMA have been recently considered, in numerous works, as appropriate candidates enabling to de- ploy the MA technique in a 5G context, [5]-[9]. Ding et al. [5] studied PD-NOMA-based systems to evaluate their user pairing-related abilities. By pairing users who enjoy a good channel situation with those suffering from poor channel conditions, overall throughput of the system may be improved. Hanif et al. [6] proposed a multi-user, multiple-input multiple-output (MIMO) PD-NOMA-based system by relying the joint power allocation and preceding design. They proposed a method for achieving the maximum system sum rate. It has been reported in many recent works that wireless transfers of power from natural sources may be achieved, and numerous advancements concerning this technique have been reported [10]. For example, multi-user communication scenarios were recommended in emerging communication systems in order to implement the Internet of Things (IoT) and fifth-generation (5G) networks [11], [12].

The benefits of energy harvesting were reported in [13]-[20]. In paper [13], benefits for the operation of wireless networks, stemming from the wireless power transfer method were described. This work proposed a new expression for achieving optimal throughput in energy-aware cooperative systems with a general time-power energy harvesting protocol, namely time-power switching-based relaying (TPSR). In particular, the impact that relay node and destination node hardware imperfections exert on two-way relaying networks (TWRN) was shown. Interestingly, to maximize system throughput, an optimized policy for joint wireless information and energy transfers was determined by identifying optimal time switching and power splitting fractions.

In [16], the authors presented a small-cell network operating in the context of heterogeneous cellular networks, for both downlink (DL) and uplink (UL) scenarios, relying on three techniques, namely energy harvesting, full-duplex transmission mode, and the power domain-based NOMA scheme. Compared to the conventional half-duplex orthogonal multiple accesses (OMA) scheme that has been widely implemented in current wireless communication systems, the full-duplex (FD) NOMA relying on an energy harvest- 
ing scheme offers great potential in terms of a further enhancement of the system's performance and has additional advantages, such as spectral efficiency, connectivity-related capabilities, and outage-related performance. In [18], the authors studied a simultaneous wireless information and power transfer for a NOMA network, with the relay being an energy-constrained device. The relay harvests energy from source radio frequency (RF) signals using the timeswitching protocol. Both imperfect channel state information (ICSI) and residual hardware impairments (RHIs) were considered. To characterize these effects in the network under consideration, outage probability (OP) and throughputrelated expressions were designed.

Motivated by the recent publications [13]-[20], we consider, in this study, a power beacon (PB) helping the access point (AP) transmit signal at the downlink portion of a NOMA system.

The remaining part of this paper is organized as follows. Section 2 introduces the principle of a downlink NOMA and describes how the signal may be processed and detected at each of the receivers. Section 3 provides an analysis of outage probability and some useful insights. Section 4 presents the results of simulations with two users, thus allowing us to confirm some of the comparisons made. A summary is provided in Section 5.

Table 1

Key parameters of the system model

\begin{tabular}{|c|l|}
\hline Symbols & Description \\
\hline$a_{i}$ & Power allocation coefficient \\
\hline$P_{S}$ & Transmit power at AP \\
\hline$P_{P}$ & Transmit power at PB \\
\hline $\bar{x}_{i}$ & Information of $U_{i}$ \\
\hline$R_{i}$ & Target rate at $U_{i}$ \\
\hline$T$ & $\begin{array}{l}\text { Total time used for energy harvesting } \\
\text { and information processing }\end{array}$ \\
\hline$\eta$ & Energy harvesting efficiency \\
\hline$\theta$ & Time switching factor \\
\hline
\end{tabular}

\section{System Model}

In Fig. 1, the AP is equipped with multiple antennas, i.e. $N$ to guarantee the operation of such an AP on the downlink, while the PB transfers wireless energy to the AP. The AP transmits the superimposed signal to destinations $U_{1}$, $U_{2}$ which will receive signal $x_{S}=\sqrt{a_{1} P_{S}} x_{1}+\sqrt{a_{2} P_{S}} x_{2}$, in which $a_{1}$ and $a_{2}$ are power allocation factors. The condition applying to the factors is that $a_{1}+a_{2}=1$, with $a_{2}>a_{1}$.

During the energy harvesting phase, the index of the best antenna may be determined and the energy received from the signal at the AP may be expressed as follows:

$$
n^{*}=\arg \underbrace{\max }_{n=1,2, \ldots, N} \bar{X}, \bar{X} \in\left\{\left|h_{0, n}\right|^{2},\left|h_{n, 1}\right|^{2},\left|h_{n, 2}\right|^{2}\right\},
$$

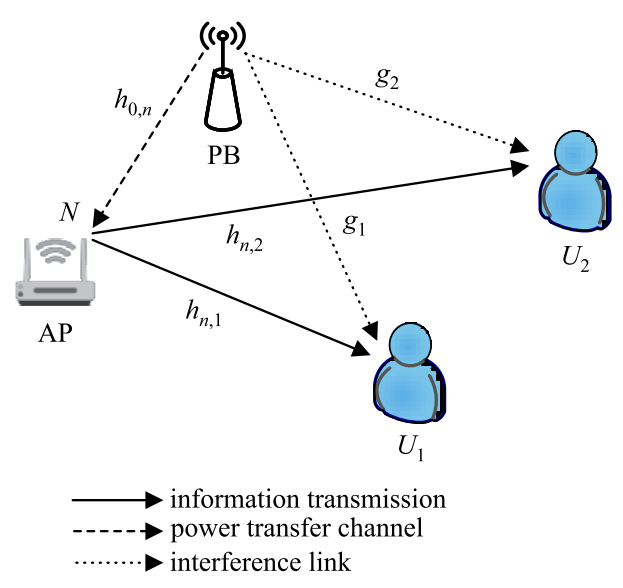

Fig. 1. Model of a power beacon-based downlink NOMA system.

and

$$
y_{S}^{n}=\sqrt{P_{P}} h_{0, n} x_{S}+\omega_{S}^{n},
$$

where $P_{P}$ is the transmit power at the $\mathrm{PB}, h_{0, n}$ denotes the power transfer channel with $h_{0, n} \sim C N\left(0, \lambda_{0}\right), x_{S}$ is the energy signal vector satisfying the total power constraint $\mathbb{E}\left\{x_{1}^{2}\right\}=\mathbb{E}\left\{x_{2}^{2}\right\}=1$ in which $\mathbb{E}\{$.$\} is the expectation op-$ erator and $\omega_{S}^{n}$ denotes the additive white Gaussian noise (AWGN) with $\omega_{S}^{n} \sim C N\left(0, N_{0}\right)$.

In this model, the power-splitting energy harvesting technique is employed [13]. Hence, the total harvested energy at the end of the first phase is:

$$
E=\eta P_{P} \theta T\left|h_{0, n}\right|^{2},
$$

where $T$ is the block time in which a certain amount of information is transmitted from the AP node to two user nodes, $\theta$ is the fraction of the block time in which the AP harvests energy from the PB's information signal and $\eta(0<\eta<1)$ denotes the energy conversion efficiency.

In line with reports from several previous papers, we assume that all of the harvested energy is used during the information transmission phase. Hence, the transmit power of the source is:

$$
P_{S}=\frac{E}{(1-\theta) T}=\frac{\eta P_{P} \theta\left|h_{0, n}\right|^{2}}{1-\theta} .
$$

During the wireless information transfer (WIT) phase, the AP employs the harvested energy to convey independent signals, serving multiple users through the NOMA paradigm.

In this case, the PB uses the beamforming technique in order to enhance performance. As such, the signal received by two users may be written as:

$$
y_{U_{i}}^{n}=h_{n, i}\left(\sqrt{a_{1} P_{S}} \bar{x}_{1}+\sqrt{a_{2} P_{S}} \bar{x}_{2}\right)+\sqrt{P_{P}} g_{i} x_{S}+\omega_{U_{i}}^{n},
$$

where $h_{n, i}, i \in\{1,2\}$ denotes the main channel from AP to $U_{i}$ in which $h_{n, 1} \sim C N\left(0, \lambda_{1}\right)$ and with $h_{n, 2} \sim C N\left(0, \lambda_{2}\right)$, while $g_{i}$ denotes interference from the PB to two users. 
The elements of $h_{n, i}$ and $g_{i}$ are independent and identically distributed zero-mean complex Gaussian random variables with variance $\lambda_{i}$ and $\Omega_{i}$, respectively. $\bar{x}_{i}$ denotes the source symbol with unit power with $\mathbb{E}\left\{\bar{x}_{i}^{2}\right\}=1$. $x_{S}$ is the jamming signal with unit power. Here, $\omega_{U_{i}}^{n}$ is the AWGN at user with variance $N_{0}$. Therefore, the received signal-to-interferenceplus-noise ratio (SINR) at $U_{1}$ to detect $U_{2}$ message $x_{2}$ is given by:

$$
\begin{aligned}
\gamma_{U_{2} \rightarrow U_{1}} & \simeq \frac{a_{2} P_{S}\left|h_{n^{*}, 1}\right|^{2}}{a_{1} P_{S}\left|h_{1, n^{*}}\right|^{2}+P_{P} \Omega_{1}+N_{0}} \\
& \simeq \frac{\varphi_{2}\left|h_{0, n^{*}}\right|^{2}\left|h_{n^{*}, 1}\right|^{2}}{\varphi_{1}\left|h_{0, n^{*}}\right|^{2}\left|h_{n^{*}, 1}\right|^{2}+\Theta_{1}},
\end{aligned}
$$

where $\rho=\frac{P_{P}}{N_{0}}$ is the transmit signal-to-noise ratio (SNR) at the source. $\varphi_{1}=\frac{a_{1} \eta \rho \theta}{(1-\theta)}, \varphi_{2}=\frac{a_{2} \eta \rho \theta}{(1-\theta)}$ and $\Theta_{1}=\rho \Omega_{1}+1$. We assume that $\mathbb{E}\left\{\left|g_{i}\right|^{2}\right\} \approx \Omega_{i}, i \in\{1,2\}$.

After SIC, the received SINR at $U_{1}$ detecting its own message $x_{1}$ is:

$$
\gamma_{U_{1}} \simeq \frac{\varphi_{1}\left|h_{0, n^{*}}\right|^{2}\left|h_{n^{*}, 1}\right|^{2}}{\Theta_{1}} .
$$

The received signal at $U_{2}$ is $y_{U_{2}}$ in Eq. (5), and the SINR at $U_{2}$ is represented by:

$$
\gamma_{U_{2}} \simeq \frac{\varphi_{2}\left|h_{0, n^{*}}\right|^{2}\left|h_{n^{*}, 2}\right|^{2}}{\varphi_{1}\left|h_{0, n^{*}}\right|^{2}\left|h_{n^{*}, 2}\right|^{2}+\Theta_{2}},
$$

where $\Theta_{2}=\rho \Omega_{2}+1$.

\section{Outage Probability Analysis}

\subsection{Outage Probability of $U_{1}$}

According to the NOMA protocol, the complementary events of an outage at $U_{1}$ can be explained by the fact that $U_{1}$ may detect $x_{2}$ as well as its own message $x_{1}$. From the above description, the outage probability of $U_{1}$ can be defined as:

$$
\begin{aligned}
\mathcal{O P}_{1} & =\operatorname{Pr}\left(\gamma_{U_{2} \rightarrow U_{1}}<\gamma_{t h 2} \cup \gamma_{U_{1}}<\gamma_{t h 1}\right) \\
& =1-\operatorname{Pr}\left(\gamma_{U_{2} \rightarrow U_{1}}>\gamma_{t h 2}, \gamma_{U_{1}}>\gamma_{t h 1}\right) \\
& =1-\operatorname{Pr}\left(\left|h_{0, n^{*}}\right|^{2}\left|h_{n^{*}, 1}\right|^{2}>\chi\right)
\end{aligned}
$$

where the threshold SNRs are $\gamma_{t h 1}=2^{\frac{R_{1}}{1-\theta}}-1, \gamma_{t h 2}=2^{\frac{R_{2}}{1-\theta}}-$ 1 in which $R_{i}$ is the target rate at $U_{i}, \delta_{1}=\frac{\Theta_{1} \gamma_{t h 1}}{\varphi_{1}}, \delta_{2}=$ $\frac{\gamma_{t h 2} \Theta_{1}}{\varphi_{2}-\varphi_{1} \gamma_{t h 2}}$ and $\chi=\max \left(\delta_{1}, \delta_{2}\right)$.

Before computing the outage probability, we assume that all channel models are followed by Rayleigh fading. The probability density function (PDF) of channel gains $\bar{X}$, $\bar{X} \in\left\{\left|h_{0, n}\right|^{2},\left|h_{n, 1}\right|^{2},\left|h_{n, 2}\right|^{2}\right\}$, i.e. $f_{\bar{X}^{*}}(x)$ is given by [22, Eq. (6)]:

$$
f_{X^{*}}(x)=\sum_{n=1}^{N}\left(\begin{array}{c}
N \\
n
\end{array}\right) \frac{n(-1)^{n-1}}{\lambda_{\bar{X}}} \mathrm{e}^{-\frac{n x}{\lambda_{\bar{X}}}} .
$$

Proposition 1: The following PDF of $f_{\left|h_{0, n^{*}}\right|^{2}\left|h_{n^{*}, i}\right|^{2}}, i \in$ $\{1,2\}$ is calculated as:

$$
\begin{aligned}
& f_{\left|h_{0, n^{*}}\right|^{2}\left|h_{n^{*}, i}\right|^{2}}(x)=\sum_{n_{0}=1}^{N} \sum_{n_{i}=1}^{N}\left(\begin{array}{c}
N \\
n_{i}
\end{array}\right)\left(\begin{array}{c}
N \\
n_{0}
\end{array}\right) \\
& \times \frac{n_{i}(-1)^{n_{i}+n_{0}-2}}{\lambda_{i}}\left[\frac{\lambda_{i}}{n_{i}}-2 \sqrt{\frac{n_{0} \lambda_{i} x}{\lambda_{0} n_{i}}} K_{1}\left(2 \sqrt{\frac{n_{0} n_{i} x}{\lambda_{0} \lambda_{i}}}\right)\right],
\end{aligned}
$$

where $K_{n}(x)$ is the first-order modified Bessel function of the second kind.

\section{Proof 1: See Appendix A.}

Proposition 2: The closed-form expression of approximated $\mathcal{O P}_{1}$ can be represented as:

$$
\begin{aligned}
\mathcal{O P}_{1} \approx & 1-\sum_{n_{0}=1}^{N} \sum_{n_{1}=1}^{N}\left(\begin{array}{c}
N \\
n_{1}
\end{array}\right)\left(\begin{array}{c}
N \\
n_{0}
\end{array}\right)(-1)^{n_{1}+n_{0}-2} \\
& +\sum_{n_{0}=1}^{N} \sum_{n_{1}=1}^{N}\left(\begin{array}{c}
N \\
n_{1}
\end{array}\right)\left(\begin{array}{c}
N \\
n_{0}
\end{array}\right) \frac{n_{1}(-1)^{n_{1}+n_{0}-2}}{\lambda_{1}} \\
& \times \frac{\pi^{2}}{2 K} \sum_{k=1}^{K} \sqrt{1-\xi_{k}^{2}} \sec ^{2}\left(\frac{\left(\xi_{k}+1\right) \pi}{4}\right) \Upsilon\left(\xi_{k}\right) .
\end{aligned}
$$

where $\xi_{k}=\cos \left(\frac{\pi(2 k-1)}{2 K}\right), \Xi(a)=\tan \left(\frac{(a+1) \pi}{4}\right)+\chi$ and $\Upsilon(a)=\sqrt{\frac{n_{0} \lambda_{1} \Xi(a)}{\lambda_{0} n_{1}}} K_{1}\left(2 \sqrt{\frac{n_{0} n_{1} \Xi(a)}{\lambda_{0} \lambda_{1}}}\right)$.

Proof 2: See Appendix B.

\subsection{Outage Probability of $U_{2}$}

In a similar way, the outage probability at the second user is computed as:

$$
\begin{aligned}
\mathcal{O P} & =1-\operatorname{Pr}\left(\gamma_{U_{2}}>\gamma_{t h 2}\right) \\
& =1-\operatorname{Pr}\left(\left|h_{0, n^{*}}\right|^{2}\left|h_{n^{*}, 2}\right|^{2}>\bar{\delta}_{2}\right),
\end{aligned}
$$

where $\bar{\delta}_{2}=\frac{\gamma_{t h 2} \Theta_{2}}{\varphi_{2}-\varphi_{1} \gamma_{t h 2}}$.

Similarly to the manner in which $\mathcal{O P}_{1}$ was solved, the closeform expression of approximated $\mathcal{O P}_{2}$ may be computed as:

$$
\begin{aligned}
& \mathcal{O P}_{2} \approx 1-\sum_{n_{0}=1}^{N} \sum_{n_{2}=1}^{N}\left(\begin{array}{c}
N \\
n_{2}
\end{array}\right)\left(\begin{array}{c}
N \\
n_{0}
\end{array}\right)(-1)^{n_{2}+n_{0}-2} \\
& +\sum_{n_{0}=1}^{N} \sum_{n_{2}=1}^{N} \sum_{q=1}^{Q}\left(\begin{array}{c}
N \\
n_{2}
\end{array}\right)\left(\begin{array}{c}
N \\
n_{0}
\end{array}\right) \frac{\sqrt{1-\xi_{q}^{2}} n_{2} \pi^{2}}{2 Q \lambda_{2}} \\
& \times(-1)^{n_{2}+n_{0}-2} \Theta\left(\xi_{q}\right) \sec ^{2}\left(\frac{\left(\xi_{q}+1\right) \pi}{4}\right)
\end{aligned}
$$

where $\xi_{q}=\cos \left(\frac{\pi(2 q-1)}{2 Q}\right), \Phi(a)=\left[\tan \left(\frac{(a+1) \pi}{4}\right)+\bar{\delta}_{2}\right]$ and $\Theta(a)=\sqrt{\frac{n_{0} \lambda_{2} \Phi(a)}{\lambda_{0} n_{2}}} K_{1}\left(2 \sqrt{\frac{n_{0} n_{2} \Phi(a)}{\lambda_{0} \lambda_{2}}}\right)$. 


\subsection{Approximate Expression of Outage Probability for Two Users}

Based on the analytical results presented in Eq. (14) and Eq. (12), when $\rho \rightarrow \infty$, the approximate outage probabilities of two users with $\chi \approx 0$ and $\bar{\delta}_{2} \approx 0$ are given as:

$$
\begin{aligned}
& \mathcal{O P}_{1}^{\infty} \approx 1-\sum_{n_{0}=1}^{N} \sum_{n_{1}=1}^{N}\left(\begin{array}{c}
N \\
n_{1}
\end{array}\right)\left(\begin{array}{c}
N \\
n_{0}
\end{array}\right)(-1)^{n_{1}+n_{0}-2} \\
& \times\left[1-\frac{n_{1} \pi^{2}}{2 \lambda_{1} K} \sum_{k=1}^{K} \sqrt{1-\xi_{k}^{2}} \sec ^{2}\left(\frac{\left(\xi_{k}+1\right) \pi}{4}\right)\right. \\
& \left.\times \sqrt{\frac{n_{0} \lambda_{1} \hat{\Xi}\left(\xi_{k}\right)}{\lambda_{0} n_{1}}} K_{1}\left(2 \sqrt{\frac{n_{0} n_{1} \hat{\Xi}\left(\xi_{k}\right)}{\lambda_{0} \lambda_{1}}}\right)\right]
\end{aligned}
$$

and

$$
\begin{aligned}
& \mathcal{O P}_{2}^{\infty} \approx 1-\sum_{n_{0}=1}^{N} \sum_{n_{2}=1}^{N}\left(\begin{array}{c}
N \\
n_{2}
\end{array}\right)\left(\begin{array}{c}
N \\
n_{0}
\end{array}\right)(-1)^{n_{2}+n_{0}-2} . \\
& \times\left[1-\frac{n_{2} \pi^{2}}{2 \lambda_{2} Q} \sum_{q=1}^{Q} \sqrt{1-\xi_{q}^{2}} \sec ^{2}\left(\frac{\left(\xi_{q}+1\right) \pi}{4}\right)\right. \\
& \left.\times \sqrt{\frac{n_{0} \lambda_{2} \hat{\Xi}\left(\xi_{q}\right)}{\lambda_{0} n_{2}}} K_{1}\left(2 \sqrt{\frac{n_{0} n_{2} \hat{\Xi}\left(\xi_{q}\right)}{\lambda_{0} \lambda_{2}}}\right)\right],
\end{aligned}
$$

where $\hat{\Xi}(a)=\left[\tan \left((\alpha+1) \frac{\pi}{4}\right)+1\right]$.

Next, in order to obtain more insights, diversity orders for $U_{1}$ and $U_{2}$ are investigated. In particular, they may be defined as [15, Eq. (18)]

$$
d_{a}=-\underbrace{\lim }_{\rho \rightarrow \infty} \frac{\log \left(\mathcal{O P} \mathcal{P}_{a}^{\infty}\right)}{\log (\rho)}, a \in\{1,2\} .
$$

By substituting Eqs. (16) and (15) into Eq. (17), the diversity orders of $U_{1}$ and $U_{2}$ are given as:

$$
d_{1}=d_{2}=0
$$

Remark. From Eq. (18) at high SNR, the outage probability of $U_{1}$ and $U_{2}$ approaches a fixed non-zero constant, indicating that outage performance error floors exist.

\subsection{Throughput for Two Users}

Based on achievable outage probability, throughput in the delay-limited transmission mode may be evaluated as $[16$, Eq. (42)]:

$$
\tau_{\star}=\left(1-\mathcal{O} \mathcal{P}_{\star}\right) R_{\star} \quad, \star \in\{1,2\}
$$

\section{Ergodic Capacity for Two Users}

In this section, the ergodic capacity is further metric evaluated at $U_{2}$ as [23]:

$$
\mathcal{C}_{2}=(1-\theta) \mathbb{E}\left[\log _{2}\left(1+\gamma_{U_{2}}\right)\right]
$$

Proposition 3: The closed-form expression for ergodic rate of $\mathcal{C}_{2}$ is given by:

$$
\begin{aligned}
& \mathcal{C}_{2}=\sum_{n_{0}=1}^{N} \sum_{n_{2}=1}^{N}\left(\begin{array}{c}
N \\
n_{0}
\end{array}\right)\left(\begin{array}{c}
N \\
n_{2}
\end{array}\right) \frac{(1-\theta)(-1)^{n_{0}+n_{2}-2}}{\ln 2} \\
& \times\left[G_{1,3}^{3,1}\left(\begin{array}{l|c}
\left.\bar{\Psi} \mid \begin{array}{c}
0 \\
0,1,0
\end{array}\right)-G_{1,3}^{3,1}
\end{array}\left(\begin{array}{l|c}
\tilde{\Psi} & 0 \\
0,1,0
\end{array}\right)\right],\right.
\end{aligned}
$$

where $G_{p, q}^{m, n}[$.$] is the Meijer G-function given in [21, Eq.$ (9.301)], $\bar{\Psi}=\frac{n_{2} n_{0} \Theta_{2}}{\lambda_{2} \lambda_{0}\left(\varphi_{2}+\varphi_{1}\right)}$ and $\tilde{\Psi}=\frac{n_{2} n_{0} \Theta_{2}}{\lambda_{2} \lambda_{0} \varphi_{1}}$.

Proof 3: See Appendix $C$.

Next, the ergodic capacity at $U_{1}$ is given as:

$$
\begin{aligned}
\mathcal{C}_{1} & =(1-\theta) \mathbb{E}\left[\log _{2}\left(1+\gamma_{U_{1}}\right)\right] \\
& =(1-\theta) \mathbb{E}\left[\log _{2}(1+\underbrace{\frac{\varphi_{1}\left|h_{0, n^{*}}\right|^{2}\left|h_{n^{*}, 1}\right|^{2}}{\Theta_{1}}}_{X})\right] \\
& =\frac{(1-\theta)}{\ln 2} \int_{0}^{\infty} \frac{1-F_{X}(x)}{1+x} \mathrm{~d} x .
\end{aligned}
$$

Based on the formula (31) from appendix $\mathrm{C}, F_{X}(x)$ is:

$$
\begin{aligned}
F_{X}(x)= & -\operatorname{Pr}\left(\left|h_{0, n^{*}}\right|^{2}\left|h_{n^{*}, 1}\right|^{2}>\frac{\Theta_{1}}{\varphi_{1}} x\right) \\
= & 1-2 \sum_{n_{0}=1}^{N} \sum_{n_{1}=1}^{N}\left(\begin{array}{c}
N \\
n_{0}
\end{array}\right)\left(\begin{array}{c}
N \\
n_{1}
\end{array}\right)(-1)^{n_{0}+n_{1}-2} \\
& \times \sqrt{\frac{n_{2} n_{0} \Theta_{1} x}{\lambda_{2} \lambda_{0} \varphi_{1}}} K_{1}\left(2 \sqrt{\frac{n_{2} n_{0} \Theta_{1} x}{\lambda_{2} \lambda_{0} \varphi_{1}}}\right) .
\end{aligned}
$$

By replacing Eq. (23) with Eq. (22) and using [21, Eq. (7.811.5), (9.34.3)], $\mathcal{C}_{1}$ may be defined as:

$$
\begin{aligned}
\mathcal{C}_{1}= & \sum_{n_{0}=1}^{N} \sum_{n_{1}=1}^{N}\left(\begin{array}{c}
N \\
n_{0}
\end{array}\right)\left(\begin{array}{c}
N \\
n_{1}
\end{array}\right) \frac{(1-\theta)(-1)^{n_{0}+n_{1}-2}}{\ln 2} \\
& \times G_{1,3}^{3,1}\left(\frac{n_{2} n_{0} \Theta_{1}}{\lambda_{2} \lambda_{0} \varphi_{1}} \mid \begin{array}{c}
0 \\
0,1,0
\end{array}\right) .
\end{aligned}
$$

\section{Numerical Results}

In this section, we simulate some theoretical results illustrated by specific figures in order to show the outage performance of the system. The key parameters are presented in Table 2. Additionally, the Gauss-Chebyshev parameter is selected as $K=Q=100$ to yield a close approximation. Figure 2 shows an improvement in outage performance at high transmit SNR. One may notice that lower outage probability rates may be achieved at SNR greater than $40 \mathrm{~dB}$. The outage performance of the second user outperforms that of the first user. The higher the number of antennas at the AP, the better the outage behavior. In particular, performance is the best for the second user. It is further confirmed that the approximated outage performance is very close to the exact value at high SNR. The differences concerning 
Table 2

Definition of system parameters [25]

\begin{tabular}{|l|c|c|}
\hline \multicolumn{1}{|c|}{ Parameter } & Notation & Values \\
\hline Power splitting factors & $a_{1}, a_{2}$ & $0.1,0.9$ \\
\hline $\begin{array}{l}\text { Target SINR rates to } \\
\text { decode } x_{1} \text { and } x_{2}\end{array}$ & $R_{1}=R_{2}$ & 0.5 \\
\hline Energy conversion efficiency & $\eta$ & 0.8 \\
\hline Fraction of the block time & $\theta$ & 0.2 \\
\hline & $\lambda_{0}$ & 1 \\
Exponential distribution & $\lambda_{1}$ & 0.4 \\
random variables & $\lambda_{2}$ & 0.6 \\
& $\Omega_{1}=\Omega_{2}$ & 0.01 \\
\hline
\end{tabular}

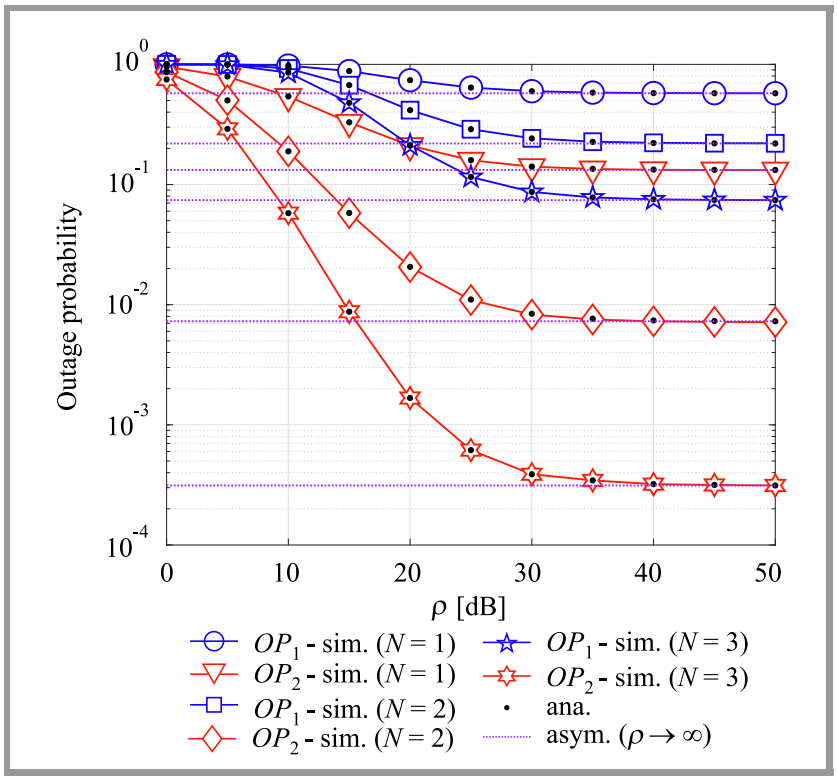

Fig. 2. Outage probability versus transmit SNR at AP with varying number of transmit antennas.

performance of two users exist throughout the entire SNR range. This is cause primarily by the fact that different power allocation factors are assigned to the individual users. In order to consider the impact that interference from the PB has on the two users, the outage probability of NOMA in the downlink mode is shown in Fig. 3. The performance of NOMA improves remarkably with the different power levels of interference channels. This is a promising result, as lower interference boosts outage performance. These outage trends for two users are similar to the trend shown in Fig. 2. Consequently, limitation of the impact of interference channels contributes to enhancing the performance of a NOMA system.

As can be seen from Fig. 4, with the increase of SNR, the outage performance of NOMA is still better than that of OMA as in Fig. 4. Interestingly, the saturation happens

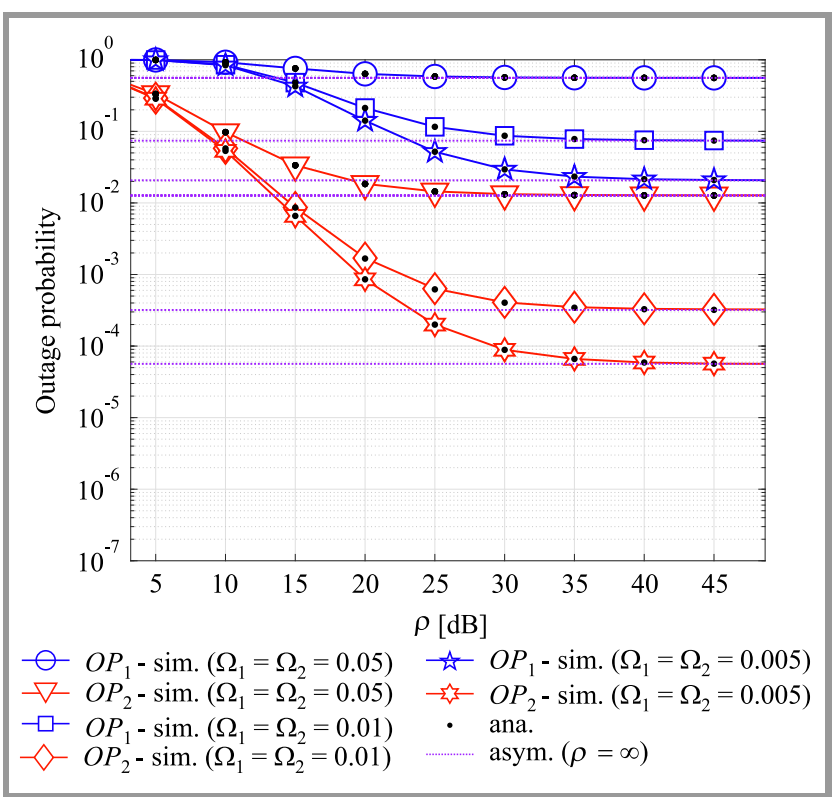

Fig. 3. Outage performance of two users with different levels of interference and $N=3$.

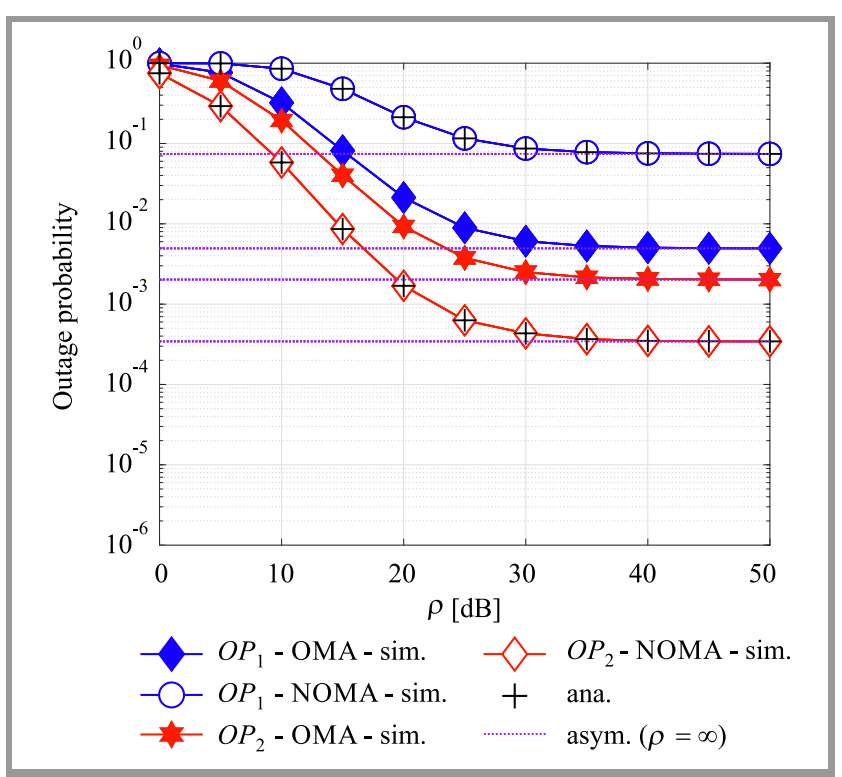

Fig. 4. Comparison between OMA and NOMA with $N=3$.

at high SNR. This result indicates that NOMA shows its superiority compared with OMA.

In Fig. 5, one may observe that a large amount of energy harvested influences the outage performance of two users. At a high region of $\theta$, higher harvested energy leads to higher SINR, then outage performance can be improved significantly. Specifically, outage performance may be changed significantly in the case of $N=3$. Therefore, a power beacon plays an important role in improving system performance.

As seen from Fig. 6, the throughput of two users increases significantly along with the increase in data rates. There are specific optimal points along these throughput performance 


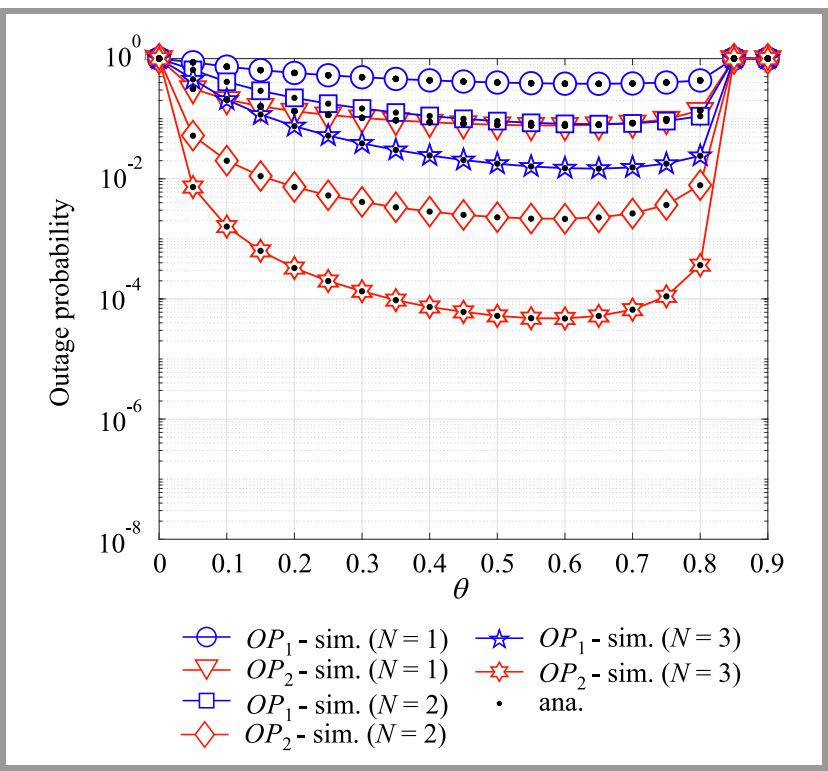

Fig. 5. Outage performance of two users vs. $\theta$, with $\rho=40 \mathrm{~dB}$.

curves. The throughput-related result is consistent with outage performance of two users shown in the previous figures.

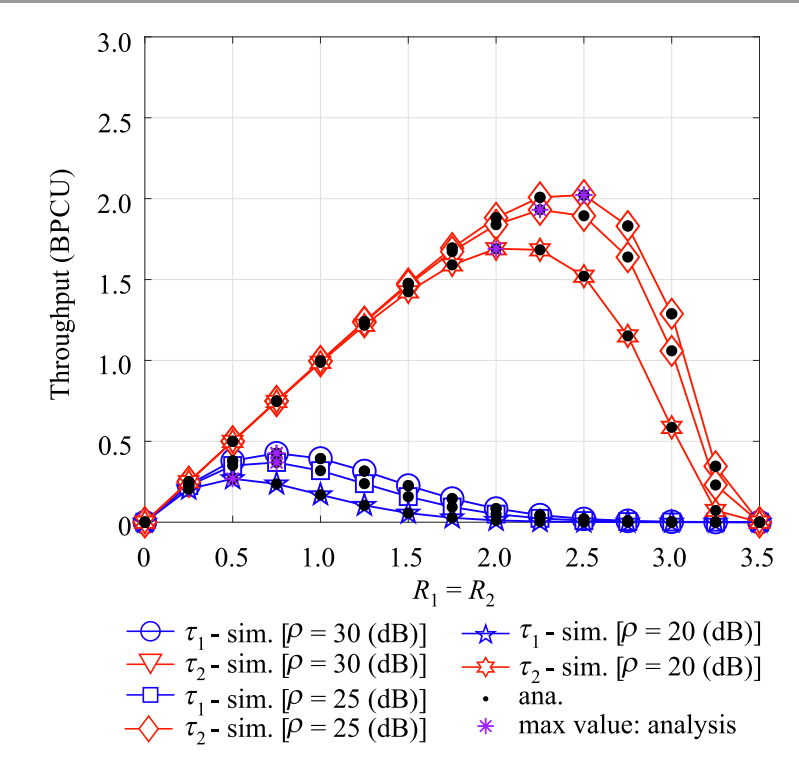

Fig. 6. Throughput performance of two users, with $N=3, a_{1}=$ $0.05, a_{2}=0.95$ and $\rho=40 \mathrm{~dB}$.

Specific ergodic capacity trends may be noticed with SNR increasing from 0 to 50, as illustrated in Fig. 7. It is obvious that high SNR rates lead to better ergodic performance, but ergodic capacity increases significantly in the low SNR range only, as demonstrated by the fact that the lines remain unchanged at high SNR rates. In the considered cases, the highest ergodic capacity may be observed when the AP is facilitated with $N=3$ antennas. This is cause by the fact that the selected antennas contribute to the improvement in ergodic capacity.

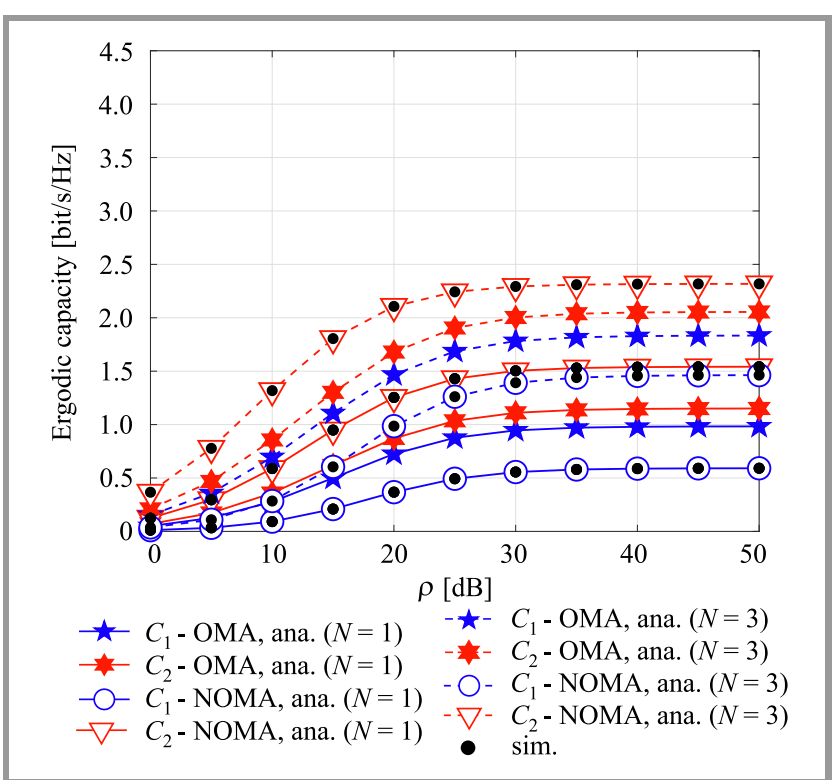

Fig. 7. Ergodic capacity versus transmit SNR.

Variations in the power splitting coefficient $\theta$ affect the ergodic capacity as well as illustrated in Fig. 8. It is worth noting that user $U_{2}$ in the NOMA mode shows superior performance for two cases $N=1,3$. This is caused mainly by the fact that SINR depends on the level of power used to transmit signals at the AP. In particular, the power splitting coefficient of $\theta$ results in higher transmit power at the AP.

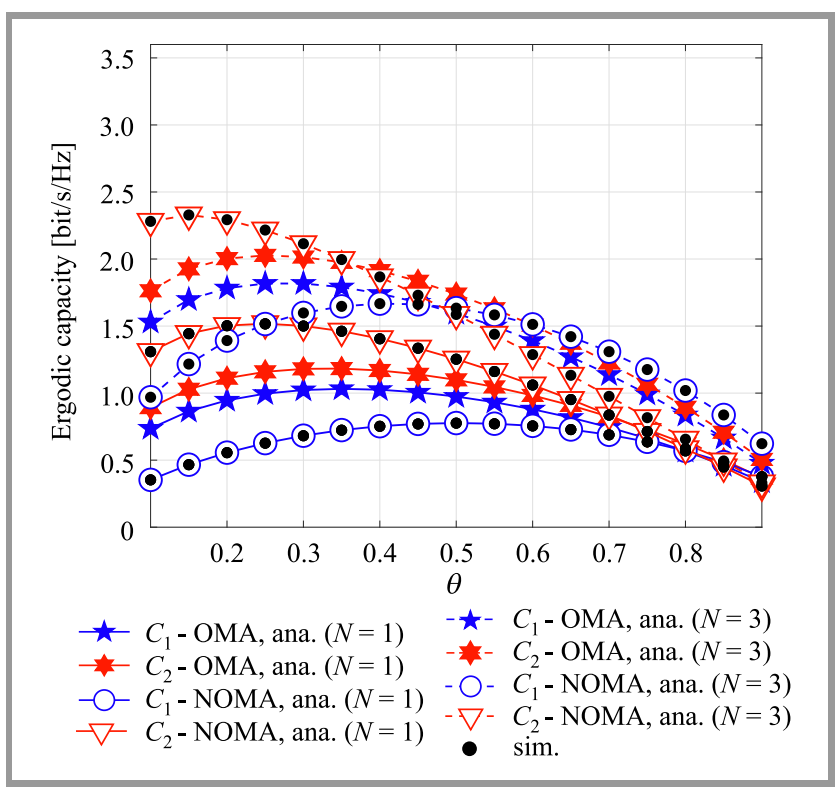

Fig. 8. Ergodic capacity versus power splitting coefficient $\theta$, with $\rho=30 \mathrm{~dB}$.

Similarly to Fig. 8, ergodic capacity may be improved once the number of transmit antennas at the AP is increased, as shown in Fig. 9. It is noteworthy that the ergodic capacity of user $U_{1}$ in the NOMA mode is likely affected by varying $N$. 


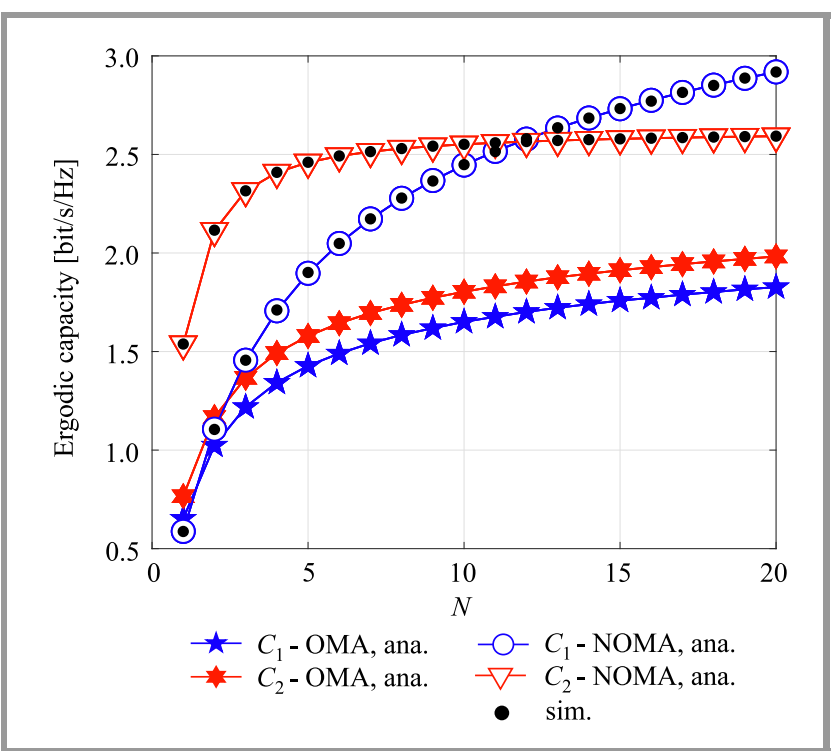

Fig. 9. Ergodic capacity versus $N$, with $\rho=30 \mathrm{~dB}$.

\section{Conclusion}

In this study, a downlink of NOMA relying on the WIT scheme was considered. Specifically, by employing energy from the $\mathrm{PB}$, the AP assists in transmitting the signal to two users in the downlink mode. Performance of the system depends on the amount of power harvested, meaning that larger numbers of AP antennas contribute to improvement in performance. Furthermore, performance of the system may be boosted by limiting the impact of interference. Finally, we have analyzed performance of the NOMA network and derived closed-form expressions for outage probability, throughput, and ergodic capacity. It has been shown that the theoretical approximations align perfectly with the simulation results. Additionally, the impact of the WIT strategy is analyzed. For example, a significant improvement is achieved in terms of outage behavior, throughput and ergodic capacity.

\section{Appendix A}

From formula (10), we have $f_{\left|h_{0, n^{*}}\right|^{2}\left|h_{n^{*}, i}\right|^{2}}(x)$ are the PDF of two random variables (RVs) $\left|h_{0, n^{*}}\right|^{2}\left|h_{n^{*}, i}\right|^{2}$ with $i \in\{1,2\}$ is calculated by:

$$
\begin{aligned}
& f_{\left|h_{0, n^{*}}\right|^{2}\left|h_{n^{*}, i}\right|^{2}}(x)=\operatorname{Pr}\left(\left|h_{0, n^{*}}\right|^{2}<\frac{x}{\left|h_{n^{*}, i}\right|^{2}}\right) \\
& =\int_{0}^{\infty} f_{\left|h_{n^{*}, i}\right|^{2}}(y) \int_{0}^{\infty} f_{\left|h_{0, n^{*}}\right|^{2}}(z) \mathrm{d} y \mathrm{~d} z \\
& =\sum_{n_{0}=1}^{N} \sum_{n_{i}=1}^{N}\left(\begin{array}{c}
N \\
n_{i}
\end{array}\right)\left(\begin{array}{c}
N \\
n_{0}
\end{array}\right) \frac{n_{i}(-1)^{n_{i}+n_{0}-2}}{\lambda_{i}}
\end{aligned}
$$

$$
\begin{aligned}
& \times \int_{0}^{\infty} \mathrm{e}^{-\frac{n_{i} y}{\lambda_{i}}}\left[1-\mathrm{e}^{-\frac{n_{0} x}{\lambda_{0} y}}\right] \mathrm{d} y \\
= & \sum_{n_{0}=1}^{N} \sum_{n_{i}=1}^{N}\left(\begin{array}{c}
N \\
n_{i}
\end{array}\right)\left(\begin{array}{c}
N \\
n_{0}
\end{array}\right) \frac{n_{i}(-1)^{n_{i}+n_{0}-2}}{\lambda_{i}} \\
& \times\left[\int_{0}^{\infty} \mathrm{e}^{-\frac{n_{i} y}{\lambda_{i}}} \mathrm{~d} y-\int_{0}^{\infty} \mathrm{e}^{-\frac{n_{i} y}{\lambda_{i}}-\frac{n_{0} x}{\lambda_{0} y}} \mathrm{~d} y\right] .
\end{aligned}
$$

Based on [14, Eq. (3.324.1)] and by applying some polynomial expansion manipulations, we obtain the statistic function of two RVs $\left|h_{0, n^{*}}\right|^{2}\left|h_{n^{*}, i}\right|^{2}$ as in Proposition 1.

\section{Appendix B}

Using the PDF from Eq. (11), the outage probability $\mathcal{O P}_{1}$ may be expressed by:

$$
\begin{aligned}
& \mathcal{O P} \mathcal{P}_{1}=1-\operatorname{Pr}\left(\left|h_{0, n^{*}}\right|^{2}\left|h_{n^{*}, 1}\right|^{2}>\chi\right) \\
& =1-\int_{\chi}^{\infty} f_{\left|h_{0, n^{*}}\right|^{2}\left|h_{n^{*}, 1}\right|^{2}}(x) \mathrm{d} x \\
& =1-\sum_{n_{0}=1}^{N} \sum_{n_{1}=1}^{N}\left(\begin{array}{l}
N \\
n_{1}
\end{array}\right)\left(\begin{array}{l}
N \\
n_{0}
\end{array}\right) \frac{n_{1}(-1)^{n_{1}+n_{0}-2}}{\lambda_{1}} \\
& \times \int_{\chi}^{\infty}\left[\frac{\lambda_{1}}{n_{1}}-2 \sqrt{\frac{n_{0} \lambda_{1} x}{\lambda_{0} n_{1}}} K_{1}\left(2 \sqrt{\frac{n_{0} n_{1} x}{\lambda_{0} \lambda_{1}}}\right)\right] \mathrm{d} x \\
& =1-\sum_{n_{0}=1}^{N} \sum_{n_{1}=1}^{N}\left(\begin{array}{c}
N \\
n_{1}
\end{array}\right)\left(\begin{array}{l}
N \\
n_{0}
\end{array}\right)(-1)^{n_{1}+n_{0}-2} \\
& +2 \sum_{n_{0}=1}^{N} \sum_{n_{1}=1}^{N}\left(\begin{array}{c}
N \\
n_{1}
\end{array}\right)\left(\begin{array}{c}
N \\
n_{0}
\end{array}\right) \frac{n_{1}(-1)^{n_{1}+n_{0}-2}}{\lambda_{1}} \\
& \times \underbrace{\int_{\chi}^{\infty} \sqrt{\frac{n_{0} \lambda_{1} x}{\lambda_{0} n_{1}}} K_{1}\left(2 \sqrt{\frac{n_{0} n_{1} x}{\lambda_{0} \lambda_{1}}}\right)}_{\mathcal{A}} \mathrm{d} x .
\end{aligned}
$$

Unfortunately, identifying a closed-form expression for $\mathcal{A}$ is a tough task, but an accurate approximation may be obtained instead. With variable $x=\tan [(t+1) \pi / 4]+\chi \Rightarrow$ $d x=\pi \sec ^{2}[(t+1) \pi / 4] / 4$ and the Gaussian-Chebyshev quadrature from [24, Eq. (25.4.38)], $\mathcal{A}$ can be represented as:

$$
\begin{aligned}
\mathcal{A}= & \frac{\pi}{4} \int_{-1}^{1} \sec ^{2}\left[\frac{(t+1) \pi}{4}\right] \sqrt{\frac{n_{0} \lambda_{1}(\tan [(t+1) \pi / 4]+\chi)}{\lambda_{0} n_{1}}} \\
& \times K_{1}\left(2 \sqrt{\frac{n_{0} n_{1}(\tan [(t+1) \pi / 4]+\chi)}{\lambda_{0} \lambda_{1}}}\right) \mathrm{d} t \\
\approx & \frac{\pi^{2}}{K 4} \sum_{k=1}^{K} \sqrt{1-\xi_{k}^{2}} \sec ^{2}\left[\frac{\left(\xi_{k}+1\right) \pi}{4}\right] \Upsilon\left(\xi_{k}\right)
\end{aligned}
$$


where $\sec ^{2}(t)=\frac{1}{\cos ^{2}(t)}, \quad \xi_{k}=\cos \left(\frac{\pi(2 k-1)}{2 K}\right), \quad \Xi(t)=$ $\tan \left[\frac{(t+1) \pi}{4}\right]+\chi$ and $\Upsilon(t)=\sqrt{\frac{n_{0} \lambda_{1} \Xi(t)}{\lambda_{0} n_{1}}} K_{1}\left[2 \sqrt{\frac{n_{0} n_{1} \Xi(t)}{\lambda_{0} \lambda_{1}}}\right]$.

Combining Eq. (27) into Eq. (26), $\mathcal{O P}_{1}$ can be identified by:

$$
\begin{aligned}
\mathcal{O P}_{1} \approx & 1-\sum_{n_{0}=1}^{N} \sum_{n_{1}=1}^{N}\left(\begin{array}{c}
N \\
n_{1}
\end{array}\right)\left(\begin{array}{c}
N \\
n_{0}
\end{array}\right)(-1)^{n_{1}+n_{0}-2} \\
& +\sum_{n_{0}=1}^{N} \sum_{n_{1}=1}^{N}\left(\begin{array}{c}
N \\
n_{1}
\end{array}\right)\left(\begin{array}{c}
N \\
n_{0}
\end{array}\right) \frac{n_{1}(-1)^{n_{1}+n_{0}-2}}{\lambda_{1}} \\
& \times \frac{\pi^{2}}{2 K} \sum_{k=1}^{K} \sqrt{1-\xi_{k}^{2}} \sec ^{2}\left[\frac{\left(\xi_{k}+1\right) \pi}{4}\right] \Upsilon\left(\xi_{k}\right) .
\end{aligned}
$$

Proof 2 is completed.

\section{Appendix C}

By the definition of the expectation operator and after integration-by-part, $\mathcal{C}_{2}$ can then be evaluated as [23], [26]:

$$
\begin{aligned}
\varrho_{2} & \triangleq(1-\theta) E\left[\log _{2}\left(1+\gamma_{U_{2}}\right)\right] \\
& =\frac{1-\theta}{\ln 2} \int_{0}^{\infty} \frac{1}{1+x}\left[1-F_{\left.\left|h_{0, n^{*}}\right|^{2}\left|h_{n^{*}, 2}\right|^{2}(x)\right] \mathrm{d} x}\right. \\
& =\frac{1-\theta}{\ln 2} \int_{0}^{\frac{\varphi_{2}}{\varphi_{1}}} \frac{1}{1+x} \bar{F}_{\left|h_{0, n^{*}}\right|^{2}\left|h_{n^{*}, 2}\right|^{2}}\left(\frac{x \Theta_{2}}{\varphi_{2}-x \varphi_{1}}\right) \mathrm{d} x,
\end{aligned}
$$

where $\bar{F}_{\left|h_{0, n^{*}}\right|^{2}\left|h_{n^{*}, 2}\right|^{2}}(x)=1-F_{\left|h_{0, n^{*}}\right|^{2}\left|h_{n^{*}, 2}\right|^{2}}(x)$. Note that $\mathrm{C}_{2}$ is derived on the condition of $\varphi_{2}-x \varphi_{1}>0$. By changing variable $t=\frac{x \Theta_{2}}{\varphi_{2}-x \varphi_{1}}$ and by performing a series of calculations, Eq. (29) can be further derived as [27]:

$$
\begin{aligned}
\varrho_{2}= & \frac{1-\theta}{\ln 2} \int_{0}^{\infty}\left(\frac{1}{t+\Theta_{2}\left(\varphi_{2}+\varphi_{1}\right)^{-1}}-\frac{1}{t+\Theta_{2} \varphi_{1}^{-1}}\right) \\
& \times \bar{F}_{\left|h_{0, n^{*}}\right|^{2}\left|h_{n^{*}, 2}\right|^{2}}\left(\frac{x \Theta_{2}}{\varphi_{2}-x \varphi_{1}}\right) \mathrm{d} t .
\end{aligned}
$$

In Eq. (29), $F_{\left|h_{0, n^{*}}\right|^{2}\left|h_{n^{*}, 2}\right|^{2}}(x)$ is calculated as:

$$
\begin{aligned}
& F_{\left|h_{0, n^{*}}\right|^{2}\left|h_{n^{*}, 2}\right|^{2}}(x)=1-\operatorname{Pr}\left(\left|h_{0, n^{*}}\right|^{2}\left|h_{n^{*}, 2}\right|^{2}>t\right) \\
& =1-\sum_{n_{0}=1}^{N} \sum_{n_{2}=1}^{N}\left(\begin{array}{c}
N \\
n_{0}
\end{array}\right)\left(\begin{array}{c}
N \\
n_{2}
\end{array}\right) \frac{n_{0}(-1)^{n_{0}+n_{2}-2}}{\lambda_{0}} \\
& \times \int_{0}^{\infty} \mathrm{e}^{-\frac{n_{2} t}{\lambda_{2} y}-\frac{n_{0} y}{\lambda_{0}}} \mathrm{~d} y \\
& =1-2 \sum_{n_{0}=1}^{N} \sum_{n_{2}=1}^{N}\left(\begin{array}{l}
N \\
n_{0}
\end{array}\right)\left(\begin{array}{l}
N \\
n_{2}
\end{array}\right)(-1)^{n_{0}+n_{2}-2} \\
& \times \sqrt{\frac{n_{2} n_{0} t}{\lambda_{2} \lambda_{0}}} K_{1}\left(2 \sqrt{\frac{n_{2} n_{0} t}{\lambda_{2} \lambda_{0}}}\right) .
\end{aligned}
$$

By substituting Eq. (31) into Eq. (30), $\mathcal{C}_{2}$ can be given by:

$$
\begin{aligned}
\mathcal{C}_{2}= & \sum_{n_{0}=1}^{N} \sum_{n_{2}=1}^{N}\left(\begin{array}{c}
N \\
n_{0}
\end{array}\right)\left(\begin{array}{c}
N \\
n_{2}
\end{array}\right) \frac{(1-\theta)(-1)^{n_{0}+n_{2}-2}}{\ln 2} \\
& \times \int_{0}^{\infty}\left(\frac{1}{t+\Theta_{2}\left(\varphi_{2}+\varphi_{1}\right)^{-1}}-\frac{1}{t+\Theta_{2} \varphi_{1}^{-1}}\right) \\
& \times 2 \sqrt{\frac{n_{2} n_{0} t}{\lambda_{2} \lambda_{0}}} K_{1}\left(2 \sqrt{\frac{n_{2} n_{0} t}{\lambda_{2} \lambda_{0}}}\right) \mathrm{d} t .
\end{aligned}
$$

Finally, with the help of [21, Eq. (7.811.5), (9.34.3)] and after some manipulations, we can obtain (21).

Proof 3 is completed.

\section{References}

[1] Q. C. Li, H. Niu, A. T. Papathanassiou, and G. Wu, "5G network capacity: Key elements and technologies", IEEE Vehicular Technol. Mag., vol. 9, no. 1, pp. 71-78, 2014 (DOI: 10.1109/MVT.2013.2295070).

[2] Y. Saito, A. Benjebbour, Y. Kishiyama, and T. Nakamura, "System level performance evaluation of downlink non-orthogonal multiple access (NOMA)", in Proc. IEEE 24th Annual Int. Symp. on Personal, Indoor, and Mobile Radio Commun. (PIMRC), London, UK, 2013. pp. 611-615 (DOI: 10.1109/PIMRC.2013.6666209).

[3] D.-T. Do, A.-T. Le, C.-B. Le, and B. M. Lee, "On exact outage and throughput performance of cognitive radio based non-orthogonal multiple access networks with and without D2D link", Sensors, vol. 19, no. 15, pp. 3314, 2019 (DOI: 10.3390/s19153314).

[4] D.-T. Do and M.-S. Van Nguyen, "Device-to-device transmission modes in NOMA network with and without wireless power transfer", Computer Commun., vol. 139, pp. 67-77, 2019

(DOI: 10.1016/j.comcom.2019.04.003).

[5] Z. Ding, P. Fan, and H. V. Poor, "Impact of user pairing on $5 \mathrm{G}$ nonorthogonal multiple-access downlink transmissions", IEEE Trans. Veh. Technol, vol. 65, no. 8, pp. 6010-6023, 2016 (DOI: 10.1109/TVT.2015.2480766).

[6] M. F. Hanif, Z. Ding, T. Ratnarajah, and G. K. Karagiannidis, "A minorization-maximization method for optimizing sum rate in the downlink of non-orthogonal multiple access systems", IEEE Trans. Signal Process, vol. 64, no. 1, pp. 76-88, 2016 (DOI: 10.1109/TSP.2015.2480042).

[7] D.-T. Do, M. Vaezi, and T.-L. Nguyen, "Wireless powered cooperative relaying using NOMA with imperfect CSI", in Proc. of IEEE Globecom Workshops, Abu Dhabi, UAE, pp. 1-6, 2018 (DOI: 10.1109/GLOCOMW.2018.8644154).

[8] D.-T. Do and A.-T. Le, "NOMA based cognitive relaying: Transceiver hardware impairments, relay selection policies and outage performance comparison", Computer Commun., vol. 146, pp. 144-154, 2019 (DOI: 10.1016/j.comcom.2019.07.023).

[9] D.-T. Do, A.-T. Le, and B.-M. Lee, "On performance analysis of underlay cognitive radio-aware hybrid OMA/NOMA networks with imperfect CSI", Electronics, vol. 8, no. 7, pp. 819, 2019 (DOI: 10.3390/electronics8070819).

[10] P.-M. Nam, D.-T. Do, T.-T. Nguyen, and P. T. Tin, "Energy harvesting assisted cognitive radio: random location-based transceivers scheme and performance analysis", Telecommun. Systems, vol. 67, no. 1, pp. 123-132, 2018 (DOI: 10.1007/s11235-017-0325-0).

[11] T.-L. Nguyen and Dinh-Thuan Do, "Exploiting impacts of intercell interference on SWIPT-assisted non-orthogonal multiple access", Wireless Commun. and Mobile Comput., vol. 2018, 2018 (DOI: 10.1155/2018/2525492).

[12] D.-T. Do, M.-S. Van Nguyen, T.-A. Hoang, and M. Voznak, "NOMA-assisted multiple access scheme for IoT deployment: Relay selection model and secrecy performance improvement, Sensors, vol. 19, no. 3, pp. 736, 2019 (DOI: 10.3390/s19030736). 
[13] D.-T. Do, "Energy-aware two-way relaying networks under imperfect hardware: optimal throughput design and analysis", Telecommun. Systems, vol. 62, no. 2, pp. 449-459, 2016 (DOI: $10.1007 / \mathrm{s} 11235-015-0085-7)$.

[14] X. Li et al., "Effective rate of MISO systems over $\kappa-\mu$ shadowed fading channels", in IEEE Access, vol. 5, pp. 10605-10611, 2017 (DOI: 10.1109/ACCESS.2017.2705018).

[15] X. Yue et al., "Exploiting full/half-duplex user relaying in NOMA systems", IEEE Trans. Commun., vol. 66, no. 2, pp. 560-575, 2018 (DOI: 10.1109/TCOMM.2017.2749400).

[16] D.-T. Do, C.-B. Le, and F. Afghah, "Enabling full-duplex and energy harvesting in uplink and downlink of small-cell network relying on power domain based multiple access", IEEE Access, vol. 8, pp. 142772-142784, 2020 (DOI: 10.1109/ACCESS.2020.3013912).

[17] X. Li, J. Li, Y. Liu, Z. Ding, and A. Nallanathan, "Residual transceiver hardware impairments on cooperative NOMA networks", IEEE Transac. on Wireless Commun., vol. 19, no. 1, pp. 680-695, 2020 (DOI: 10.1109/TWC.2019.2947670).

[18] X. Li, J. Li, and L. Li, "Performance analysis of impaired SWIPT NOMA relaying networks over imperfect Weibull channels", in IEEE Systems J., vol. 14, no. 1, 2020, pp. 669-672 (DOI: 10.1109/JSYST.2019.2919654).

[19] X. Li, M. Liu, C. Deng, P. T. Mathiopoulos, Z. Ding, and Y. Liu, "Full-duplex cooperative NOMA relaying systems with I/Q imbalance and imperfect SIC", IEEE Wireless Commun. Letters, vol. 9, no. 1, pp. 17-20, 2020 (DOI: 10.1109/LWC.2019.2939309).

[20] Xingwang Li et al., "A unified framework for HS-UAV NOMA network: performance analysis and location optimization", IEEE Access, vol. 8, pp. 13329-13340, 2020 (DOI: 10.1109/ACCESS.2020.2964730).

[21] I. S. Gradshteyn and I. M. Ryzhik, Table of Integrals, Series and Products, 6th ed. New York, NY, USA: Academic Press, 2000 (ISBN: 9780080542225).

[22] C.-B. Le and D.-T. Do, "On outage performance of backscatter NOMA relaying systems equipping with multiple antennas", Electronics Letters, vol. 55, no. 19, pp. 1066-1067, 2019 (DOI: 10.1049/el.2019.1390).

[23] A. A. Nasir, X. Zhou, S. Durrani, and R. A. Kennedy, "Relaying protocols for wireless energy harvesting and information processing", IEEE Transac. on Wireless Commun., vol. 12, no. 7, pp. 3622-3636, 2013 (DOI: 10.1109/TWC.2013.062413.122042).

[24] M. Abramowitz and I. A. Stegun, Handbook of Mathematical Functions with Formulas, Graphs, and Mathematical Tables. New York, NY, USA: Dover, 1972 (ISBN: 9780486612720 )

[25] P. Yan, J. Yang, M. Liu, J. Sun, and G. Gui, "Secrecy outage analysis of transmit antenna selection assisted with wireless power beacon", IEEE Transac. on Vehicular Technol., vol. 69, no. 7, pp. 7473-7482, 2020 (DOI: 10.1109/TVT.2020.2992766).

[26] X. Wang, M. Jia, I. W.-H. Ho, Q. Guo, and F. C. M. Lau, "Exploiting fullduplex two-way relay cooperative non-orthogonal multiple access", IEEE Transac. Commun., vol. 67, no. 4, pp. 2716-2729, 2019 (DOI: 10.1109/TCOMM.2018.2890264).

[27] T.-L. Nguyen,, C.-B. Le, and D.-T. Do. "Performance analysis of multi-user NOMA over $\alpha-\kappa-\mu$ shadowed fading", Electronics Letters, vol. 56, no. 15, pp. 771-773, 2020 (DOI: 10.1049/el.2019.4265).

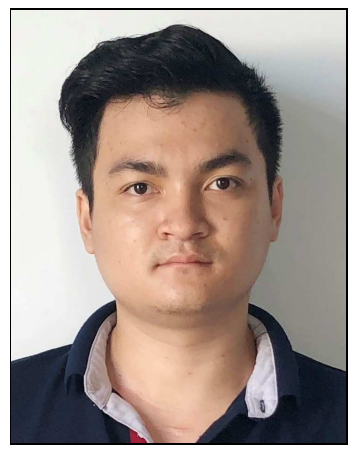

Chi-Bao Le has been working closely with Dr. Thuan of the Wireless Communications and Signal Processing Research Group at Industrial University of Ho Chi Minh City, Vietnam. He is currently pursuing his M.Sc. degree in wireless communications. His research interests include electronic design, signal processing in wireless communications network, non-orthogonal multiple access, UAV, backscatter communication, physical layer security and reconfigurable intelligent surfaces.

E-mail: lechibao@iuh.edu.vn

Faculty of Electronics Technology

Industrial University of Ho Chi Minh City

Ho Chi Minh City

Vietnam

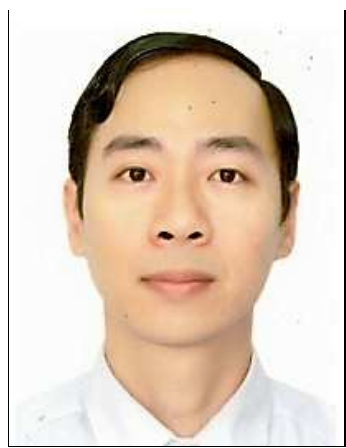

Nhan Duc Nguyen received his M.Eng. in electronic materials from International Training Institute for Materials Science (ITIMS), Hanoi University of Technology in 1998, and his Ph.D. degree in electrical and computer systems engineering from Monash University, Australia in 2011. He joined the Faculty of Telecommunications, Postal and Telecommunications Institute of Technology in Vietnam, as a lecturer, in 1999. He served as the Head of the Signals and Systems Department at Postal and Telecommunications Institute of Technology from 2014 to 2020. He is currently serving as a Systems Engineering Director at the Innovation Center, Van Lang University. His research interests focus on optical communications, numerical modeling and analysis, signal processing, as well as sensor data processing in machine learning.

E-mail: nhan.nd@vlu.edu.vn

Innovation Center

Van Lang University

Ho Chi Minh City

Vietnam 